\title{
Effects of knowledge of results and signal regularity on vigilance performance*
}

\author{
JOEL S. WARM, BILLY D. EPPS and ROBERT P. FERGUSON \\ University of Cincinnati, Cincinnati, Ohio 45221
}

\begin{abstract}
According to Baker (1963), knowledge of results (KR) facilitates vigilance performance by enhancing the generation of accurate expectations regarding the time of appearance of critical signals. The implications of this notion were assessed by comparing effects of true and false (noncontingent) KR within the context of temporally regular and irregular signal schedules. The Ss listened for increments in the duration of recurrent white-noise pulses during a 1-h vigil. Response times (RTs) to signal detections were faster and less variable when signals were presented on a regular as compared to an irregular basis. RTs were also faster and less variable for Ss receiving true or false KR relative to controls who experienced no feedback. However, the facilitative effects of true KR did not exceed those of false KR even when signal presentations were regular in time and apparently predictable. The results are considered as contrary to an expectancy interpretation of the role of KR in the vigilance task.
\end{abstract}

A consistent finding in vigilance experiments is that knowledge of results (KR) enhances overall performance efficiency and tends to slow the rate at which performance declines with time on task (Davies \& Tune, 1969). Baker (1963) has attempted to incorporate the beneficial effects of KR within the framework of expectancy theory. This position maintains that the $\mathrm{S}$ acts as a temporal averaging instrument in the monitoring task and forms expectancies concerning future signal occurrences on the basis of his experience with the task. The S's readiness to detect a signal is positively related to his level of expectancy. According to Baker, KR improves the S's expectancies regarding the time course of critical events by helping him to learn the temporal pattern of the stimuli.

Baker's interpretation of the effects of $\mathrm{KR}$ is challenged by studies using false feedback (KR not contingent with S's responses) which presumably cannot yield accurate information about the temporal distribution of critical signals. These investigations have shown that false $\mathrm{KR}$ is also effective in improving monitoring performance (Antonelli \& Karas, 1967; Loeb \& Schmidt, 1963; Mackworth, 1964; Weidenfeller, Baker, \& Ware, 1962). Moreover, with one exception (Mackworth, 1964), the effects of false KR were as efficacious as those of true KR. Such findings have led to the conclusion that the facilitative effects of feedback in vigilance are predominantly motivational rather than instructive in nature (Loeb \& Schmidt, 1963; Smith, 1966).

\footnotetext{
*This research was sponsored in part by the Institute of Space Sciences of the University of Cincinnati under National Aeronautics and Space Administration Grant NGL-36-004-014 and by the Department of Health, Education, and Welfare in conjunction with the Cincinnati Center for Developmental Disorders through Project Nos. 912 and 918. Requests for Disorders through Project Nos. Psychology, University of Cincinnati, Cincinnati, Ohio 45221
}

As described by Baker (1963), the formation of accurate expectancies regarding the temporal structure of a series of signals is inversely related to the S's uncertainty as to when a signal will appear. One means of increasing such uncertainty is through nonuniformity in the distribution of intersignal intervals (ISIs-the temporal interval between critical signals). In fairness to Baker, it is worth noting that all of the experiments which have compared true and false KR used highly irregular distributions of ISIs. The uncertainty associated with such schedules could have served to prevent effective learning of the temporal pattern of signals and thereby masked the role of an instructional component in feedback. The facilitative effects of true KR may exceed those of false KR under conditions more conducive to the formation of accurate temporal expectancies, such as the use of regular and predictable ISIs. Indeed, the fact that vigilance performance is generally more effective when regular as compared to irregular ISIs are used has been cited as primary support for the applicability of an expectancy model to this task (Davies \& Tune, 1969). The present study was designed, therefore, to examine the effects of true and false KR within the context of regular as well as irregular signal presentations.

\section{METHOD}

Seventy-two undergraduates, 31 men and 41 women, served as Ss. They ranged in age from 17 to 34 , with a mean of 20 year. None had previous experience in a vigilance study. All Ss participated in 1 -h session during which they listened for occasional increments in the duration of recurrent white-noise pulses. Acoustic stimulation was presented binaurally via headphones at an intensity of $78 \mathrm{~dB}$ SPL. Throughout the session, 0.5 -sec pulses of stimulation were presented once every 
Table 1

Response Times (in Seconds) for Three Feedback Conditions During Three Periods of Watch With Regular and Irregular Intersignal Intervals

\begin{tabular}{|c|c|c|c|c|c|c|c|c|c|}
\hline \multirow[b]{3}{*}{$\begin{array}{c}\text { Feedback } \\
\text { Conditions }\end{array}$} & \multicolumn{9}{|c|}{ Periods of Watch $(20 \mathrm{Min})$} \\
\hline & \multicolumn{3}{|c|}{1} & \multicolumn{3}{|c|}{2} & \multicolumn{3}{|c|}{3} \\
\hline & $\begin{array}{l}\text { Regular } \\
\text { ISI }\end{array}$ & $\begin{array}{l}\text { Irregular } \\
\text { ISI }\end{array}$ & Mean & $\begin{array}{l}\text { Regular } \\
\text { ISI }\end{array}$ & $\begin{array}{l}\text { Irregular } \\
\text { ISI }\end{array}$ & Mean & $\begin{array}{l}\text { Regular } \\
\text { ISI }\end{array}$ & $\begin{array}{l}\text { Irregular } \\
\text { ISI }\end{array}$ & Mean \\
\hline True KR & .52 & .57 & .54 & .45 & .60 & .52 & .42 & .65 & .54 \\
\hline False KR & .57 & .57 & .57 & .48 & .54 & .51 & .44 & .52 & .48 \\
\hline No KR & .74 & .71 & .72 & .74 & .82 & .78 & .73 & .81 & .77 \\
\hline Mean & .61 & .62 & & .56 & .65 & & .53 & .66 & \\
\hline
\end{tabular}

$2.0 \mathrm{sec}$. These constituted nonsignal events to which no overt response was to be made by S. A 1.0-sec pulse (twice the normal duration) was the critical signal to which $S$ was required to respond by depressing a pushbutton switch. The response switch was mounted in a flat-black panel and located on a table approximately $71 \mathrm{~cm}$ from the seated $S$. In order to reduce variability due to motor movement time, all Ss were instructed to keep the index finger of their preferred hand on a small white dot immediately below the response switch.

Three feedback conditions (true-KR, false-KR, and no-feedback control) were combined factorially with two ISI distributions (regular and irregular) to provide a total of six experimental groups. Twelve Ss were assigned at random to each group. The vigil was divided into three 20 -min periods, within which 12 critical signals were presented for all groups. The vigil proceeded from one period to the next without interruption. Under the regular ISI condition, critical signals occurred once very $100 \mathrm{sec}$. Intersignal intervals under the irregular ISI condition were distributed as follows: $30,45,45,60,60,60,90$, $120,120,120,150$, and $300 \mathrm{sec}(\overline{\mathrm{X}}=100 \mathrm{sec})$. In the irregular condition, this sequence of ISIs was randomly ordered for each $\mathrm{S}$ during each period of watch.

Knowledge of results was provided by a console adjacent to the panel housing the response switch. The console contained three lamps (red, amber, and green) arrayed along a horizontal vector. Feedback was given in terms of whether each response was faster, and therefore better (green), or slower and therefore worse (red), than the immediately preceding response. In the case of false KR, feedback bore no systematic relation to actual performance. "Faster" and "slower" evaluations were given on a random basis after each response, with the restriction of equal distribution within a given period. Only the amber lamp was displayed for Ss who received no evaluative information. This lamp was flashed to acknowledge each response and served as a control for the arousing properties of added stimulation associated with the visual signaling of KR.

The occurrence of neutral pulses and critical signals was controlled by solid state programming equipment and a punched tape timer. Response times (RTs) to correct detections were read to the nearest $0.01 \mathrm{sec}$ from a Standard Electric Model S-1 timer which was activated $0.5 \mathrm{sec}$ after the onset of a pulse comprising a critical event and terminated by S's response or after a no-response duration of $2.0 \mathrm{sec}$. Failure to respond to a critical signal within $2.0 \mathrm{sec}$ was considered as an error of omission (missed signal). Each $\mathrm{S}$ was tested individually in a sound-shielded room; the control equipment was located in an adjacent room. The Ss surrendered their watches at the start of the session and they had no knowledge of the length of the vigil other than it would not exceed $2 \mathrm{~h}$.

\section{RESULTS}

Two measures of performance were obtained from the data of each S: (a) the median RTs to correct detections $^{1}$ and (b) the standard deviations of RTs to correct detections.

\section{Response Time}

Means of median RTs are presented in Table 1 for the factorial combinations of feedback, time on watch, and ISI.

An analysis of variance of the data of Table 1 revealed that signals were detected more rapidly when regular as compared to irregular ISIs were used $(\mathrm{F}=6.38$, $\mathrm{df}=1 / 66, \mathrm{p}<.025)$ and that there were significant differences associated with the conditions of feedback $(\mathrm{F}=25.05, \mathrm{df}=2 / 66, \mathrm{p}<.001)$. The interaction of primary interest in this study, that between the ISI schedule and feedback, failed to reach significance $(F=1.09, \mathrm{df}=2 / 66, \mathrm{p}>.05)$, indicating that the effects associated with KR were unrelated to the temporal regularity of signal presentations. The overall effect for periods of watch also lacked significance $(F=1.20$, $\mathrm{df}=2 / 132, \quad \mathrm{p}>.05)$, but there were significant interactions between the ISI schedule and periods $(\mathrm{F}=$ 14.30, $\mathrm{df}=2 / 132, \mathrm{p}<.001)$ and between feedback and periods $(\mathrm{F}=6.30, \mathrm{df}=4 / 132, \mathrm{p}<.001)$. The second order interaction between the three factors in the analysis failed to reach significance $(F<1)$.

The nature of the ISI by Periods interaction can be determined by comparing the column means for each period in Table 1. It is evident in the table that RTs tended to decrease with time on watch when regular ISIs were used and to increase over time when the ISIs were irregular. Examination of the means for the three feedback groups in Table 1 reveals that, during each period of watch, RTs for the true-KR and false-KR groups were quite similar to each other and faster than those for the control group. The table also reveals differences in the performance trends over time for the three feedback groups. A deterioration in performance efficiency over time is reflected in the data of the control group in terms of an increment in the latency. of detections as the vigil progressed. By contrast, RTs remained relatively stable across periods for the true-KR group and became more rapid with time on watch in the false-KR group. Tests for the simple main effects of periods within the three feedback conditions revealed significant differences among periods in the control 
group $(\mathrm{F}=4.04, \mathrm{df}=2 / 132, \mathrm{p}<.025)$ and in the false-KR group $(\mathrm{F}=9.52, \mathrm{df}=2 / 132, \mathrm{p}<.001)$ but not in the true-KR group $(\mathrm{F}<1)$.

\section{Response Variability}

The standard deviations of the RTs for the three orthogonal factors (feedback, periods, and ISI schedule) were also subjected to an analysis of variance. The analysis indicated that response variability was less under regular as compared to irregular signal presentation (mean standard deviation scores $=.14$ and $.19 \mathrm{sec}$, respectively; $F=10.51, \mathrm{df}=1 / 66, \mathrm{p}<.005$ ). Significant differences in the variability of RTs were also noted between the three feedback conditions $(F=8.88$, $\mathrm{df}=2 / 66, \mathrm{p}<.001)$. The mean standard deviation scores for the truc-KR, false-KR, and control groups were $.14, .15$, and .21 , respectively. A Newman-Keuls test with an alpha level of .05 for all comparisons indicated that performance was less variable in the true- and false-KR groups relative to the controls and the true-KR and false-KR groups did not differ significantly from each other. No significant changes in response variability were noted across periods of watch (mean standard deviation scores for Periods 1-3 were $.17, .16$ and $.16 \mathrm{sec}$, respectively, $\mathrm{F}<1$ ) and all of the interactions in the analysis lacked significance $(\mathrm{p}>.05)$.

\section{DISCUSSION}

The results of this study are in only partial agreement with the implications of an expectancy model of vigilance. The finding that RTs to signal detections tended to decrease over time in the context of regular ISIs and to increase when irregular ISIs were used supports the applicability of an expectancy model. On the other hand, the data offer no support for the notion, derived from the expectancy position, that the facilitative effects of KR reside in the ability of feedback to aid Ss in learning the temporal pattern of stimuli. Both true and false KR enhanced the speed of signal detections. However, even when signals were perfectly regular in time and apparently increasingly predictable with time on watch, the facilitative effects of true KR failed to exceed those of false KR. In other words, the effects associated with feedback were independent of the S's experimentally produced uncertainty regarding critical signal appearances and, presumably, his expectancies of the time course of signal presentations. In this sense, the present results are consistent with those of an earlier experiment by Wiener (1963) which also yielded a null relation between KR and temporal uncertainty when uncertainty (or expectancy) was manipulated through variations in critical signal frequency. It seems clear from this, and from the earlier investigation, that the effects of feedback are not mediated by expectancy formation. As indicated previously, enhancement of vigilance performance by false KR has led to the conclusion that the role of feedback is primarily motivational in nature. The results of this study lend further encouragement to such an explanation.

As in previous investigations (Davies \& Tune, 1969), KR was found to attenuate the deterioration of performance efficiency with time on watch that is typical in vigilance tasks. There was however, a puzzling aspect to the data: The speed of signal detections remained stable over time when KR accurately reflected the S's performance but became faster over time when noncontingent feedback was used. The manner in which positive (green light) and negative (red light) evaluations were given represents a potential source of uncontrolled variation in this study. Both types of evaluations were programmed to occur equally often in each period of watch under false KR, while their frequencies were free to vary in accord with S's performance in the case of true KR. Consequently, it might be argued that progressive disparities in the relative frequency of positive and negative evaluations may be responsible for the difference in the trends of performance over time between the true-KR and the false-KR groups. Inspection of the data revealed, however, that during each period of watch positive and negative evaluations occurred almost equally often in the true-KR condition. A difference in the temporal course of performance between trueand false-KR conditions of the sort observed in this investigation has not been reported previously. Such a difference may reflect a Type-I error and its reliability should be assessed in future experiments.

It is important to realize that explanations of monitoring performance on the basis of expectancy and motivational constructs are, to some degree, incomplete. Both types of accounts imply enhanced readiness to detect signals, but they do not specify how such readiness is related to the manner in which Ss process information from the monitored display. In terms of a filtering (Broadbent, 1971) or an observing response (Jerison, 1970) approach, increased expectancy or motivation may lead to more consistent processing of target-relevant information. Broadbent (1971) has suggested that, when RTs are involved, more stable attending may be reflected in a lowered amount of response variability. The finding that both true and false KR as well as regularity in signal presentations were associated with reductions in the variability of RTs fits easily within this point of view.

\section{REFERENCES}

Antonelli, D. C. \& Karas, G. G. Performance on a vigilance task under conditions of true and false knowledge of results. Perceptual \& Motor Skills, 1967, 25, 129-138.

Baker, C. H. Further toward a theory of vigilance. In D. N. Buckner and J. J. McGrath (Eds.), Vigilance: A symposium. New York: McGraw-Hill, 1963, Pp. 127-170.

Broadbent, D. E. Decision and Stress. New York: Academic Press, 1971 .

Davies, D. R., \& Tune, G. S. Human vigilance performance. New York: American Elsevier, 1969.

Jerison, H. J. Vigilance, discrimination and attention. In D. I. Mostofsky (Ed.), Attention: Contemporary theory and analysis. New York: Appleton-Century-Crofts, 1970. Pp. 127-147.

Loeb, M., \& Schmidt, E. A. A comparison of the effects of different kinds of information in maintaining efficiency on an auditory monitoring task. Ergonomics, 1963, 6, 75-81.

Mackworth, J. F. The effect of true and false knowledge of results on the detectability of signals in a vigilance task. Canadian Journal of Psychology, 1964, 18, 106-117.

Smith, R. L. Monotony and motivation: A theory of vig ilance. Santa Monica, Calif: Dunlap, 1966.

Warm, J. S., \& Alluisi, E. A. Influence of temporal uncertainity and sensory modality of signals on watchkeeping performance. Journal of Experimental Psychology, 1971, 87, 303-308.

Weidenfeller, E. W., Baker, R. A., \& Ware, J. R. Effects of knowledge of results (true and false) on vigilance performance. Perceptual \& Motor Skills, 1962, 14, 211-215.

Wiener, E. L. Knowledge of results and signal rate in monitoring: A transfer of training approach. Journal of Applied Psychology, 1963, 47,214-222.

\section{NOTE}

1. Previous research with this task using acoustic signals has resulted in a very high level of detection (Warm \& Alluisi, 1971). This experiment was no exception; detection probabilities under all conditions ranged from .92 to .96 , with a mean of .93 . 